\title{
Editorial
}

\section{QbD Innovation Through Advances in PAT, Data Analysis Methodologies, and Material Characterization}

\author{
William C. Stagner ${ }^{1,3}$ and Rahul V. Haware ${ }^{2,3}$
}

Received 6 August 2019; accepted 6 August 2019; published online 22 August 2019

KEY WORDS: PAT; QbD; real-time monitoring; mixing assessment; imaging; MVA; data fusion; Brillouin light scattering; Raman spectroscopy; FT-NIR; NMR relaxometry.

It has been 15 years since the FDA issued its pharmaceutical CGMPs for the 21st-century report laying out its quality by design (QbD) and process analytical technologies (PAT) initiative (1). QbD tenets emphasize the need to understand, predict, monitor, and control the interplay of critical material attributes (CMA) and critical process parameters (CPP) on critical quality product attributes (CQPA) $(2,3)$. Over the last 15 years, significant progress has been made in developing PAT and subsequent analysis of data generated from these techniques. The United States Pharmacopeia has recently issued a Chemometrics chapter that discusses the application of qualitative and quantitative techniques and the interpretation of multivariate data (4).

PAT is an important QbD tool. Raman spectroscopy has emerged as a fast, effective, and versatile PAT real-time analyzer. A review of Raman spectroscopy as a PAT tool for real-time monitoring of pharmaceutical secondary manufacturing processes is provided (5). While the emphasis of the review presents applications of Raman spectroscopy for monitoring secondary manufacturing of solids, the article also provides examples of analytical process control of upstream active pharmaceutical ingredient (API) synthesis and crystallization and biotechnology applications such as enzymatic hydrolysis and protein production. Over the last decade or so, numerous analytical technologies have been used to assess blend uniformity and mixing dynamics. Spectroscopic, velocimetric, tomographic, and acoustic

Guest Editors: William C. Stagner and Rahul V. Haware

${ }^{1}$ Department of Pharmaceutical Sciences, Campbell University College of Pharmacy \& Health Sciences, Campbell University, Buies Creek, NC 27506, USA.

${ }^{2}$ Division of Pharmaceutical Sciences, Arnold \& Marie Schwartz College of Pharmacy, Long Island University, 75 DeKalb Ave. Rm HS 608, Brooklyn, NY 11201, USA.

${ }^{3}$ To whom correspondence should be addressed. (e-mail: stagnerw@campbell.edurahul.haware@liu.edu) emission mixing assessment techniques are reviewed (6). Use of imaging techniques as PAT tools has been steadily increasing as the accuracy, precision, speed, and robustness of these analyzers has improved, and they have become commercially available. An in-line vision imaging system is described that provides real-time mini-tab film-coat thickness estimates during fluid-bed coating (7).

Pharmaceutical applications of hot melt extrusion (HME) have been reported since the 1970s. HME has found particular promise as a scalable continuous manufacturing process to improve the solubility and bioavailability of many recent breakthrough APIs. A technical note demonstrating the application of FT-NIR analysis to provide real-time monitoring of a HME product exemplifies the utility of PAT in continuous processing (8).

Data analysis methodologies are another important QbD tool. The increased array of real-time process analyzers used to monitor any given process or material generates very large and complex data sets. Thus, the intricacies of such complex data treatment requires sophisticated data management strategies. A timely review demonstrates the challenges presented by the current state of pharmaceutical material characterization methods and the incorporation of multivariate analysis to enhance the understanding of pharmaceutical materials (9). Data fusion is a relatively new and powerful data analysis technique. Some of the first pharmaceutical applications were reported in early 2000. Data fusion is a methodology that combines multiple data sources to obtain a more informed, accurate, consistent, robust, and sophisticated understanding. For the first time, critical process parameters and near-infrared (NIR) spectroscopy data are employed to predict final product dissolution that could be used for realtime release testing (10). Data fusion of 5 critical process parameters and 4 principal component analysis NIR scores were used to develop a predictive partial least squares regression model. The data fusion method incorporates the NIR chemical (coating concentration; water concentration) and physical (coat thickness) properties with the processing 
parameters. The data fusion technique provided significantly improved predictions compared to individual spectral models.

Material characterization is a fundamental component of QbD. Since the publication of FDA's QbD initiative, there has been a significant increase in the development and application of material sensors. Brillouin light scattering (BLS) may be the next novel PAT developed. BLS theory was developed nearly 100 years ago and is broadly used to determine the mechanical properties of any material phase. Pharmaceutical applications include fundamental understanding of structure-function relationships to direct crystal engineering, anisotropy and its effect on deformation behavior, and phase behavior of materials. Currently, there are no commercial BLS spectrophotometers available. However, individual components can be assembled with advanced technology interferometers that are designed for improved signal stability and accurate BLS recordings. The improved interferometer design has led to a renaissance of BLS applications such as the mechanical characterization of thin films, semisolids, powders, and biological cells and organelles. An informative BLS review is included that presents an overview and history of the technology, instrumental design, BLS theory and key outputs, current pharmaceutical applications, and near term applications (11). A research article highlights the novel ability of BLS to provide nascent aggregate powder elasticity data. The aggregate elasticity and tabletability of 25 compounds exhibiting a wide range of intermolecular forces was used to validate the BLS approach (12). New drug delivery technologies can expand the need for innovative sensors to allow real-time characterization and analysis. PATs capable of physicochemical evaluation and monitoring of the manufacture of robust nanoparticle drug delivery systems are lagging. A research article that describes the characterization of a nanostructured lipid carrier is provided as an example of some of the analytical methods that require creative analyzer approaches to implement realtime PAT (13). In 2015, the FDA approved the first 3Dprinted drug dosage form for the U.S. market. The approval has led to a surge of interest in ink-jet printing, which offers many manufacturing advantages such as continuous processing, precise and accurate dosing, and flexibility and ease of achieving multiple doses. The biggest potential impact of inkjet printing lies in the possibility of accelerating personalized medicine. This novel dosage form also expands the need to develop PAT technologies for real-time monitoring and release. Raman and NIR quantitation of three ink-jet printed drugs on a porous substrate is reported (14). The results indicate that the two analytical methods provide fast and accurate API content, either alone or possibly in combination. The innovative use of benchtop NMR relaxometry is reported for the nondestructive quantitation of a FlexPen ${ }^{\circledR}$ insulin suspension product. The results support the possible development of a nondestructive novel PAT capable of quality assessment of each injection device (15).

This theme issue is dedicated to QbD innovation through advances in PAT, data analysis methodologies, and material characterization. The aim of this theme is to inform pharmaceutical scientists about some of the QbD innovations occurring in the pharmaceutical sciences, which can be applied to meet ICH and regulatory $\mathrm{QbD}$ expectations for manufacturing economical and built-in quality pharmaceutical products. New drug delivery technologies continue to require PAT and sensor innovation. BLS may be the next major breakthrough pharmaceutical material characterization and PAT method.

\section{REFERENCES}

1. FDA 2004. Pharmaceutical CGMPs for the 21st century - a risk-based approach, final report. U.S. Department of Health and Human Services. Food and Drug Administration. Sept. 2004. https://www.fda.gov/media/77391/download. Accessed on Jun 2019.

2. FDA 2004. Guidance for industry: PAT - a framework for innovative pharmaceutical development, manufacturing, and quality assurance. https://www.fda.gov/media/71012/download. Accessed Jun 2019.

3. Yu LX, Amidon G, Khan MA, Hoag SW, Polli J, Raju GK, Woodcock J. Understanding pharmaceutical quality by design. AAPS J. 2014;16(4):771-83.

4. United States Pharmacopeia. U.S.P.-N.F. online. U.S.P. 40 - N.F. 35, 2017.

5. Nagy B, Farkas A, Borbás E, Vass P, Nagy ZK, Marosi G. Raman spectroscopy for process analytical technologies of pharmaceutical secondary manufacturing. AAPS PharmSciTech. 2018;20(1):1.

6. Crouter A, Briens L. Methods to assess mixing of pharmaceutical powders. AAPS PharmSciTech. 2019;20(2):84.

7. Podrekar G, Kitak D, Mehle A, Lavrič Z, Likar B, Tomaževič $D$, Dreu R. In-line film coating thickness estimation of minitablets in a fluid-bed coating equipment. AAPS PharmSciTech. 2018;19(8):3440-53.

8. Vo AQ, He H, Zhang J, Martin S, Chen R, Repka MA. Application of FT-NIR analysis for in-line and real-time monitoring of pharmaceutical hot melt extrusion: a technical note. AAPS PharmSciTech. 2018;19(8):3425-9.

9. Ferreira AP, Gamble JF, Leane MM, Park H, Olusanmi D, Tobyn M. Enhanced understanding of pharmaceutical materials through advanced characterisation and analysis. AAPS PharmSciTech. 2018;19(8):3462-80.

10. Ibrahim A, Kothari BH, Fahmy R, Hoag SW. Prediction of dissolution of sustained release coated ciprofloxacin beads using near-infrared spectroscopy and process parameters: a data fusion approach. AAPS PharmSciTech. 2019;20(6):222.

11. Singaraju AB, Bahl D, Stevens LL. Brillouin light scattering: development of a near century-old technique for characterizing the mechanical properties of materials. AAPS PharmSciTech. 2019;20(3):109.

12. Bahl D, Singaraju AB, Stevens LL. Aggregate elasticity and Tabletability of molecular solids: a validation and application of powder Brillouin light scattering. AAPS PharmSciTech. 2018;19(8):3430-9.

13. Fahmy UA. Augmentation of fluvastatin cytotoxicity against prostate carcinoma PC3 cell line utilizing alpha lipoic-ellagic acid nanostructured lipid carrier formula. AAPS PharmSciTech. 2018;19(8):3454-61.

14. Edinger M, Iftimi L-D, Markl D, Al-Sharabi M, Bar-Shalom D, Rantanen J, Genine N. Quantification of inkjet-printed pharmaceuticals on porous substrates using Raman spectroscopy and near-infrared spectroscopy. AAPS PharmSciTech. 2019;20(5):207.

15. Briggs KT, Taraban MB, Wang W, Yu YB. Nondestructive quantitative inspection of drug products using benchtop NMR relaxometry - the case of NovoMix ${ }^{\circledR} 30$. AAPS PharmSciTech. 2019;20(5):189.

Publisher's Note Springer Nature remains neutral with regard to jurisdictional claims in published maps and institutional affiliations. 